\title{
Photons from quantized eletric flux representations
}

\author{
Madhavan Varadarajan \\ Raman Research Institute, Bangalore 560 080, India. \\ madhavan@rri.ernet.in
}

March 2001

\begin{abstract}
The quantum theory of $U(1)$ connections admits a diffeomorphism invariant representation in which the electric flux through any surface is quantized. This representation is the analog of the representation of quantum $S U(2)$ theory used in loop quantum gravity. We investigate the relation between this representation, in which the basic excitations are 'polymer-like', and the Fock representation, in which the basic excitations are wave-like photons. We show that normalizable states in the Fock space are associated with 'distributional' states in the quantized electric flux representation. This work is motivated by the question of how wave-like gravitons in linearised gravity arise from polymer-like states in non-perturbative loop quantum gravity.
\end{abstract}




\section{Introduction}

The loop quantum gravity approach [1, 2] is based on a Hamiltonian description of classical general relativity in which the basic configuration variable is a connection [3, 4] and its conjugate is a triad field. The spatial 3-metric on a Cauchy slice is constructed from the triad and the connection contains information about the extrinsic curvature of the slice as embedded in the spacetime, thus establishing contact with the usual Arnowitt-Deser-Misner formulation. In the quantum theory, the basic connection dependent operators are holonomies of the connection around loops in the Cauchy slice [5, 6].

In recent years the approach has been put on a firm mathematical footing with the following key features. A (quantum) configuration space, $\overline{\mathcal{A} / \mathcal{G}}$, of (generalised) $S U(2)$ connections modulo gauge and a canonical diffeomorphism invariant measure, $d \mu_{0}$ (also called the Ashtekar-Lewandowski measure [7]), on $\overline{\mathcal{A} / \mathcal{G}}$ have been constructed [8, 7, 9, 10, 11]. The space $L^{2}\left(\overline{\mathcal{A} / \mathcal{G}}, d \mu_{0}\right)$ provides a kinematical Hilbert space on which the (self adjoint) $S U(2)$ holonomy operators act by multiplication. This kinematical Hilbert space is spanned by an orthonormal set of 'spin network' states, each associated with an oriented, closed graph whose edges are labelled by representations of $S U(2)$ 12, 13.

Every spin network state is an eigen state of operators corresponding to the area of 2-surfaces in the Cauchy slice - roughly speaking, the area of a surface gets a

contribution of $\sqrt{j(j+1)}$ units of Planck area from each edge labelled by spin $j$ which intersects the surface transversely. Since the area operator is constructed from the triad field (or equivalently the $S U(2)$ electric field), the edges of the graph may be thought of as carrying quanta of non abelian electric flux. Since the edges are 1 dimensional, the intuitive picture of states in $L^{2}\left(\overline{\mathcal{A} / \mathcal{G}}, d \mu_{0}\right)$ is of 'polymer like' quantum excitations.

As noted earlier, $L^{2}\left(\overline{\mathcal{A} / \mathcal{G}}, d \mu_{0}\right)$ is a kinematical structure. The dynamics of general relativity in its canonical description is encoded in the diffeomorphism and Hamiltonian constraints. Physical states in the quantum theory are in the kernel of the 
corresponding quantum constraint operators. The representation of holonomies, functionals of the triad, as well as the unitary action of diffeomorphisms on $L^{2}\left(\overline{\mathcal{A} / \mathcal{G}}, d \mu_{0}\right)$ provides a representation of the diffeomorphism constraints [14], and in a key breakthrough by Thiemann, the Hamiltonian constraint [15]. It turns out that elements of the kernel of the constraints are 'too distributional' to be normalizable states in $L^{2}\left(\overline{\mathcal{A} / \mathcal{G}}, d \mu_{0}\right)$. Rather, they are expressible as non-normalizable, infinite, sums of spin network states.

Thus, a nonperturbative physical state of the quantum gravitational field is a distributional sum of kinematic states, each of which is associated with 1 dimensional, polymer-like excitations. Given such quantum states, a key open question is: how do classical configurations of the gravitational field arise ? In particular, how does flat spacetime (and small perturbations around it) arise from non-perturbative quantum states of the gravitational field?

The latter question is particularly interesting for the following reason. Small perturbations about flat spacetime correspond to solutions of linearized gravity. Quantum states of linearised gravity lie in the familiar graviton Fock space on which the conventional perturbative approaches to quantum gravity are based. Such approaches seem to fail due to nonrenormalizability problems. Thus, an understanding of the relation between the quantum states of linearised gravity and states in full nonperturbative loop quantum gravity would shed light on the reasons behind the failure of perturbative methods.

There are many aspects of this yet-to- be-understood relation between perturbative and nonperturbative states and we shall focus on only one of them, namely, the dramatically different nature of the basic excitations in Fock space and in loop quantum gravity: whereas Fock space gravitons can be thought of as propagating $3 d$ wave-packets, states in loop quantum gravity are associated with $1 d$ polymer-like excitations.

This aspect of the relation between perturbative and non perturbative states in quantum gravity can be isolated and studied in the simpler yet highly instructive context of source free Maxwell theory. To this end, consider a $U(1)$ connection on 
a spatial slice diffeomorphic to $R^{3}$. Starting from the Poisson bracket algebra of $U(1)$ holonomies around loops in $R^{3}$, it can be seen that exact counterparts of the $S U(2)$ constructions of loop quantum gravity exist [8, [7, 16, 17]. Thus, a canonical diffeomorphism invariant measure exists on the quantum configuration space of generalised $U(1)$ connections modulo gauge (we shall continue to denote these by $d \mu_{0}$ and $\overline{\mathcal{A} / \mathcal{G}}$ - it will be clear from the context whether these symbols refer to $S U(2)$ or $U(1))$, holonomies act as unitary operators by multiplication on $L^{2}\left(\overline{\mathcal{A} / \mathcal{G}}, d \mu_{0}\right)$, and $L^{2}\left(\overline{\mathcal{A} / \mathcal{G}}, d \mu_{0}\right)$ is spanned by an orthonormal basis each element of which is associated with a closed, oriented graph and a labelling of edges of the graph by representations of $U(1)$. Since such representations are labelled by integers called charges, we refer to these states as 'charge network' states. Each such state is an eigen function of electric flux operators associated with surfaces in $R^{3}$ and the edges of the graph underlying the state may be thought of as carrying quanta of electric flux. We refer to this diffeomorphism invariant representation, in which the basic excitations are, once again, 1 dimensional and 'polymer-like' as the quantized electric flux (qef) representation.

In sharp contrast to this, is the usual Poincare invariant quantum theory of a $U(1)$ connection on a fixed flat spacetime with flat spatial slices diffeomorphic to $R^{3}$. Here, the connection and its conjugate electric field are represented as operator valued distributions on the Fock space of photons where the basic quantum excitations are 3d and wave-like.

Thus quantum $U(1)$ theory presents an excellent arena to discuss the question raised earlier, namely how can the 3d wave-like excitations of Fock space arise from underlying $1 d$ polymer-like excitations?

At first sight, the Fock and the qef representations are very different. In the Fock representation, the connection is an operator valued distribution which needs to be smeared in 3 dimensions to obtain a well defined operator. Holonomies of this operator valued distribution involve smearings only over the 1 dimension provided by the loop, and are not well defined. In contrast, in the qef representation, holonomies are well defined operators on $L^{2}\left(\overline{\mathcal{A} / \mathcal{G}}, d \mu_{0}\right)$. In section 2 we review our previous results [18] which point to a way around this apparently insurmountable obstacle to 
relating the two representations.

In section 3 we present our main result, namely that states in the Fock representation are associated with distributional sums of charge network states in the qef representation. This mirrors the mathematical structure of loop quantum gravity in the sense that, there, physical states are associated with distributional sums of spin network states in the kinematic Hilbert space. Section 4 contains a discussion of our results and some concluding remarks.

We shall use units in which $\hbar=c=1$.

\section{The Issue of Smearing}

Since the abelian Poisson bracket algebra of holonomies is the primary structure from which the qef representation is obtained [17, 18], we would like to relate the Fock representation to this holonomy algebra. As noted earlier, the usual Fock representation is not a representation of the holonomy algebra because the connection operators in this representation are too singular for their holonomies to be defined. Instead, it is possible to obtain the usual Fock representation as the representation of a related algebra of 'smeared holonomies'. By using the smeared holonomy algebra as a link between the algebra of holonomies and the Fock representation we shall be able to relate the two.

In order to define the smeared holonomies we recall the following definitions from [19, 18]. $\vec{x}$ denotes Cartesian coordinates of a point on $R^{3} \cdot \mathcal{A}$ is the space of smooth $U(1)$ connections, $A_{a}(\vec{x})$, (on the trivial $U(1)$ bundle on $R^{3}$ ) whose cartesian components are functions of rapid decrease at infinity. $\mathcal{L}_{x_{0}}$ is the space of unparametrized, oriented, piecewise analytic loops on $R^{3}$ with basepoint $\overrightarrow{x_{0}}$. Composition of a loop $\alpha$ with a loop $\beta$ is denoted by $\alpha \circ \beta$. Given a loop $\alpha \in \mathcal{L}_{x_{0}}$, the holonomy of $A_{a}(x)$ around $\alpha$ is $H_{\alpha}(A):=\exp \left(i \oint_{\alpha} A_{a} d x^{a}\right)$. The holonomy can equivalently be defined as

$$
H_{\alpha}(A)=\exp i \int_{R^{3}} X_{\gamma}^{a}(\vec{x}) A_{a}(\vec{x}) d^{3} x
$$

\footnotetext{
${ }^{1}$ As noted in [16], to get an object with the dimensions of a $U(1)$ connection, we need to divide the usual magnetic potential by a parameter, $q_{0}$, which has dimensions of electric charge. This reflects in the Poisson bracket (7) and the definition (31).
} 
with

$$
X_{\gamma}^{a}(\vec{x}):=\oint_{\gamma} d s \delta^{3}(\vec{\gamma}(s), \vec{x}) \dot{\gamma}^{a},
$$

where $s$ is a parametrization of the loop $\gamma, s \in[0,2 \pi] . X_{\gamma}^{a}(\vec{x})$ is called the form factor of $\gamma$. The Gaussian smeared form factor [19] is defined as

$$
X_{\gamma_{(r)}}^{a}(\vec{x}):=\int_{R^{3}} d^{3} y f_{r}(\vec{y}-\vec{x}) X_{\gamma}^{a}(\vec{y})=\oint_{\gamma} d s f_{r}(\vec{\gamma}(s)-\vec{x}) \dot{\gamma}^{a}
$$

where

$$
f_{r}(\vec{x})=\frac{1}{(2 \pi)^{\frac{3}{2}} r^{3}} e^{\frac{-x^{2}}{2 r^{2}}} \quad x:=|\vec{x}|
$$

approximates the Dirac delta function for small $r$.

Then, the smeared holonomy is defined as

$$
H_{\gamma_{(r)}}(A)=\exp i \int_{R^{3}} X_{\gamma_{(r)}}^{a}(\vec{x}) A_{a}(\vec{x}) d^{3} x
$$

As shown in [18], the Fock representation is a representation of the Poisson bracket algebra generated by the smeared holonomies, $H_{\gamma_{(r)}}(A)$ and the electric field $E^{a}(\vec{x})$ :

$$
\begin{aligned}
\left\{H_{\gamma_{(r)}}, H_{\alpha_{(r)}}\right\} & =\left\{E^{a}(\vec{x}), E^{b}(\vec{y})\right\}=0, \\
\left\{H_{\gamma_{(r)}}, E^{a}(\vec{x})\right\} & =\frac{i}{q_{0}} X_{\gamma_{(r)}}^{a}(\vec{x}) H_{\gamma_{(r)}} .
\end{aligned}
$$

These Poisson brackets are generated from the elementary Poisson brackets

$$
\left\{A_{a}(\vec{x}), E^{b}(\vec{y})\right\}=\frac{1}{q_{0}} \delta_{a}^{b} \delta(\vec{x}, \vec{y})
$$

Here, $q_{0}$ is a parameter with the units of electric charge [16]. In the Fock representation, the smeared holonomies, $\hat{H}_{\gamma_{(r)}}$, are unitary operators and the electric field, $\hat{E}^{a}(\vec{x})$, is an operator valued distribution.

How is the above algebra involving smeared holonomies and the electric field related to the holonomy algebra? To this end we define the classical Gaussian smeared electric field $E_{r}^{a}(\vec{x})$ by

$$
E_{r}^{a}(\vec{x}):=\int d^{3} y f_{r}(\vec{y}-\vec{x}) E^{a}(\vec{y})
$$


The Poisson bracket algebra generated by the (unsmeared) holonomies and the Gaussian smeared electric field is

$$
\begin{aligned}
\left\{H_{\gamma}, H_{\alpha}\right\} & =\left\{E_{r}^{a}(\vec{x}), E_{r}^{b}(\vec{y})\right\}=0, \\
\left\{H_{\gamma}, E_{r}^{a}(\vec{x})\right\} & =\frac{i}{q_{0}} X_{\gamma_{(r)}}^{a}(\vec{x}) H_{\gamma} .
\end{aligned}
$$

Then, as is hinted by (6) and (9) and proved in detail in [18], the abstract algebraic structures underlying the Poisson bracket algebras generated by $\left(H_{\gamma_{(r)}}(A), E^{a}(\vec{x})\right)$ and $\left(H_{\gamma}(A), E_{r}^{a}(\vec{x})\right)$ are identical! In other words, $E^{a}(\vec{x})$ and $E_{r}^{a}(\vec{x})$, and $H_{\gamma_{(r)}}(A)$ and $H_{\gamma}(A)$ may be identified with the same abstract objects as far as the algebraic structure of the two Poisson bracket algebras is concerned. Therefore, any representation of the Poisson bracket algebra generated by $\left(H_{\gamma_{(r)}}(A), E^{a}(\vec{x})\right)$ defines a representation of the Poisson bracket algebra generated by $\left(H_{\gamma}(A), E_{r}^{a}(\vec{x})\right)$. In particular, the Fock representation of the $\left(H_{\gamma_{(r)}}(A), E^{a}(\vec{x})\right)$ algebra defines a representation of the $\left(H_{\gamma}(A), E_{r}^{a}(\vec{x})\right)$ algebra. We shall call this representation, the ' $r$-Fock representation' of the $\left(H_{\gamma}(A), E_{r}^{a}(\vec{x})\right)$ Poisson bracket algebra. Thus, in the $r$-Fock representation the holonomies, $\hat{H}_{\gamma}$ are well defined operators!

We can see this explicitly as follows. The Fock representation, or for that matter, any representation with a cyclic 'vacuum' state, can be reconstructed from the vacuum expectation values of the algebra of operators. Thus, we may specify the $r$-Fock representation via the vacuum expectation values of the Fock representation as follows. The Fock representation is a cyclic representation generated from the Fock vacuum, $\mid 0>$ with vacuum expectation values [

$$
\begin{aligned}
<0\left|\hat{H}_{\gamma_{(r)}}\right| 0> & =\exp \left(-\int \frac{d^{3} k}{4 q_{0}^{2} k}\left|X_{\gamma_{(r)}}^{a}(\vec{k})\right|^{2}\right) \\
<0\left|\hat{H}_{\alpha_{(r)}} \hat{E}^{a}(\vec{x}) \hat{H}_{\beta_{(r)}}\right| 0> & =\frac{X_{\beta_{(r)}}^{a}(\vec{x})-X_{\alpha_{(r)}}^{a}(\vec{x})}{2 q_{0}} \exp \left(-\int \frac{d^{3} k}{4 q_{0}^{2} k}\left|X_{\alpha \circ \beta_{(r)}}^{a}(\vec{k})\right|^{2}\right),(11
\end{aligned}
$$

where $X_{\gamma_{(r)}}^{a}(\vec{k})$ denotes the Fourier transform of $X_{\gamma_{(r)}}^{a}(\vec{x})$. This defines the $r$-Fock representation as the cyclic representation generated from the $r$-Fock 'vacuum' $\mid 0_{r}>$

\footnotetext{
${ }^{2}$ Our conventions for the Fock space representation are displayed in section 3.2
} 
with vacuum expectation values

$$
\begin{aligned}
<0_{r}\left|\hat{H}_{\gamma}\right| 0_{r}> & =\exp -\left(\int \frac{d^{3} k}{4 q_{0}^{2} k}\left|X_{\gamma_{(r)}}^{a}(\vec{k})\right|^{2}\right) \\
<0_{r}\left|\hat{H}_{\alpha} \hat{E}_{r}^{a}(\vec{x}) \hat{H}_{\beta}\right| 0_{r}> & =\frac{\left(X_{\beta_{(r)}}^{a}(\vec{x})-X_{\alpha_{(r)}}^{a}(\vec{x})\right)}{2 q_{0}} \exp \left(-\int \frac{d^{3} k}{4 q_{0}^{2} k}\left|X_{\alpha \circ \beta_{(r)}}^{a}(\vec{k})\right|^{2}\right) .(13
\end{aligned}
$$

The fact that the holonomies are well defined operators in the $r$-Fock representation allows us to related the $r$-Fock representation and the qef representation in a fairly direct manner, as we show in the next section.

To summarise: Although it is not possible to relate the qef representation with the usual Fock representation directly, it is possible to relate the qef representation with the $r$-Fock representation, since both provide representations of the Poisson bracket algebra generated by $\left(H_{\gamma}(A), E_{r}^{a}(\vec{x})\right)$.

The only remaining question is of the relation between the Fock and the $r$-Fock representations. The mathematical relation between the two, in terms of representations of two different realizations of the same algebraic structure, is clear and is true for any $r>0$. So the only question is of the physical relation between $r$-Fock and Fock representations. We argue below that for certain measurements which are of physical relevance in the context of our motivations from quantum gravity, the two representations are physically indistinguishable for sufficiently small $r$.

Since we shall phrase our argument in terms of measurements of Fourier modes, we first discuss the behaviour of Fourier modes under Gaussian smearing. Given any function $h(\vec{x})$, its Fourier transform is

$$
h(\vec{k})=\frac{1}{(2 \pi)^{\frac{3}{2}}} \int_{R^{3}} d^{3} x h(\vec{x}) e^{-i \vec{k} \cdot \vec{x}}
$$

and its Gaussian smeared version is

$$
h_{r}(\vec{x})=\int_{R^{3}} d^{3} y f_{r}(\vec{y}-\vec{x}) h(\vec{y})
$$

It follows that

$$
h_{r}(\vec{k})=e^{\frac{-k^{2} r^{2}}{2}} h(\vec{k}) .
$$


In particular we have

$$
X_{\gamma_{(r)}}^{a}(\vec{k})=e^{\frac{-k^{2} r^{2}}{2}} X_{\gamma}^{a}(\vec{k})
$$

and

$$
E_{r}^{a}(\vec{k})=e^{\frac{-k^{2} r^{2}}{2}} E^{a}(\vec{k})
$$

From (17) and (5) it follows, in obvious notation, that

$$
H_{\gamma_{(r)}}(A(\vec{k}))=H_{\gamma}\left(e^{\frac{-k^{2} r^{2}}{2}} A(\vec{k})\right)
$$

Consider measurements of quantities at or above a length scale $L$. More precisely, let the measurements be of Fourier modes, $E^{a}(\vec{k}), A_{a}(\vec{k})$ of the electric and connection fields (in, say, the Coulomb gauge) for $k \leq \frac{1}{L}$. Further, let the accuracy of the measurement process be characterised by the positive number $\delta$. If $\Delta E^{a}(\vec{k}), \Delta A_{a}(\vec{k})$ are the accuracies to which $E^{a}(\vec{k}), A_{a}(\vec{k})$ are measured, then $\delta$ is defined through $\Delta E^{a}(\vec{k})=E^{a}(\vec{k}) \delta$ and $\Delta A_{a}(\vec{k})=A_{a}(\vec{k}) \delta$. Then if $r$ is chosen small enough that the condition $1-e^{-\frac{r^{2}}{2 L^{2}}}<\delta$ holds, it follows that the measurements cannot distinguish between the modes $E^{a}(\vec{k})$ and $e^{-\frac{k^{2} r^{2}}{2}} E^{a}(\vec{k})$, and $A_{a}(\vec{k})$ and $e^{-\frac{k^{2} r^{2}}{2}} A_{a}(\vec{k})$. Thus, the measurements cannot distinguish between unsmeared fields and their Gaussian smeared counterparts for sufficiently small $r$. Since the the primary operators in the Fock and $r$-Fock representations are related by Gaussian smearing (see equations (18) and (19)), it is straightforward to see that for any state in the Fock space there exists a state in the $r$-Fock space such that the above type of measurement can never distinguish between the two. 3

We loosely interpret this statement to mean that, given measurements at some length scale performed to some finite accuracy, there is always a sufficiently small $r$ such that the $r$-Fock representation is experimentally indistinguishable from the usual Fock representation. Although for Maxwell theory the introduction of a length scale seems arbitrary, in the context of linearised gravity it is necessary to restrict attention to length scales much larger than the Planck scale or else the linearised approximation will not be physically valid.

\footnotetext{
${ }^{3}$ In the language and notation of [18], the state in the $r$-Fock space is the image of the state in the Fock space via the map $I_{r}$.
} 
Hence, for the remainder of the paper we may alter our original question to : how can the $3 d$ wave-like excitations of $r$-Fock space arise from underlying $1 d$ polymer-like excitations?

\section{3. $r$-Fock states as distributions in the qef repre- sentation}

In this section the $r$-Fock space representation is derived from the qef representation by identifying the $r$-Fock vacuum as a distributional state in the qef representation. We show that the $r$-Fock vacuum can be written as a formal non-normalizable sum of charge network states and that it resides in the algebraic dual to the space of finite linear combinations of charge network states.

In section 3.1 we briefly review the properties of charge network states as well as the definition of the algebraic dual representation. In section 3.2.1 we encode the Poincare invariance of the Fock vacuum in a relation between smeared holonomy and electric field operators. In section 3.2.2 we show how this relation implies the identification of the $r$-Fock vacuum with a distributional sum of charge network states.

\subsection{Charge network states}

Straightforward repetitions of constructions for the $S U(2)$ case of loop quantum gravity [12, 11, 10, 13] lead to the following results for $U(1)$ charge networks.

(1) The charge network states constitute an (uncountable) orthonormal spanning set in $L^{2}\left(\overline{\mathcal{A} / \mathcal{G}}, d \mu_{0}\right)$. Each such state is labelled by a closed, oriented graph whose edges carry non-trivial representations of $U(1)$. Representations of $U(1)$ are labelled by integers called 'charges' [16], hence the name 'charge network states'. $U(1)$ gauge invariance implies that the sum of charges at each vertex vanishes. We denote the normalized state labelled by the graph ' $\gamma$ ', with $N$ edges carrying the charges $\left(p_{1} . . p_{N}\right)$ as $\mid \gamma,\{p\}>$. Orthonormality implies

$$
<\alpha,\{q\} \mid \gamma,\{p\}>=\delta_{(\alpha,\{q\}),(\gamma,\{p\})}
$$

i.e. the inner product vanishes unless $\alpha=\gamma$ and $q_{i}=p_{i}, i=1 . . N$. 
(2) The electric field operator acts as

$$
\hat{E}^{a}(\vec{x})\left|\gamma,\{p\}>=\frac{X_{\gamma,\{p\}}^{a}(\vec{x})}{q_{0}}\right| \gamma,\{p\}>.
$$

Here

$$
X_{\gamma,\{p\}}^{a}(\vec{x}):=\sum_{i=1}^{N} p_{i} \int_{e_{i}} d s_{i} \delta^{3}\left(\vec{e}_{i}\left(s_{i}\right), \vec{x}\right) \dot{e}_{i}{ }^{a} .
$$

In this equation $e_{i}$ denotes the $i$ th edge of $\gamma$ and is parametrized by the parameter $s_{i}$ so that $\vec{e}_{i}\left(s_{i}\right)$ is the coordinate of the point on the edge $e_{i}$ at parameter value $s_{i}$.

The electric flux operator associated with a surface $S$ with surface normal $n_{a}$ acts as

$$
\int_{S} \hat{E}^{a} n_{a} d^{2} s\left|\gamma,\{p\}>=\frac{\sum_{i_{S}} p_{i_{S}} \kappa_{i_{S}}}{q_{0}}\right| \gamma,\{p\}>.
$$

Here the index $i_{S}$ ranges only over the edges of $\gamma$ which intersect the surface $S$. $\kappa_{i_{S}}=1$ iff $e_{i_{S}}{ }^{a} n_{a}>0, \kappa_{i_{S}}=-1$ iff ${\dot{i_{S}}}^{a} n_{a}<0$ and $\kappa_{i_{S}}=0$ iff $e_{i_{S}}{ }^{a} n_{a}=0$. This action may be derived rigorously via a regularization along the lines of [20].

From (23), every charge network state $\mid \gamma,\{p\}>$ is an eigen state of the electric flux operator and the edges of $\gamma$ may be pictured as carrying quanta of electric flux in multiples of $q_{0}^{-1}$. Although physical intuition for (and, indeed, the naming of) the qef representation arises from this property of the electric flux, the electric flux operator itself will not play a role in the considerations of this work. Instead, it is the Gaussian smeared electric field operator which we will play a key role and we now exhibit its action on charge network states.

In the notation of (3), it follows that (21) implies

$$
\hat{E}_{r}^{a}(\vec{x})\left|\gamma,\{p\}>=\frac{X_{\gamma,\{p\}_{(r)}}^{a}(\vec{x})}{q_{0}}\right| \gamma,\{p\}>.
$$

In addition to (1) and (2), the abelian nature of $U(1)$ implies the following.

(3) Every charge network state $\mid \gamma,\{p\}>$ can be obtained from the "vacuum" state $\mid \Omega>$ (i.e. the state $\left.\Omega(A)=1, \Omega \in L^{2}\left(\overline{\mathcal{A} / \mathcal{G}}, d \mu_{0}\right)\right)$ via the action of the holonomy operator around a suitably defined loop, $\beta$, so that

$$
\left|\gamma,\{p\}>=\hat{H}_{\beta}\right| \Omega>
$$


Here $\beta=\beta_{1}^{p_{1}} \circ \beta_{2}^{p_{2}} \ldots \circ \beta_{N}^{p_{N}}$ and $\beta_{i}^{p_{i}}$ denotes the loop obtained by traversing $p_{i}$ times round $\beta_{i} . \beta_{i}, i=1 . . N$ are defined by the construction (3.2) of [7] as

$$
\beta_{i}=Q\left(v_{i}^{+}\right) \circ e_{i} \circ Q\left(v_{i}^{-}\right)
$$

where $v_{i}^{ \pm}$are the vertices of $\gamma$ which constitute the beginning and end points of $e_{i}$ and $Q(v)$ is a path from the base point $\vec{x}_{0}$ to the point $v$ such that $Q(v)$ intesects $\gamma$ at most at a finite number of isolated points. It can be verified that

$$
X_{\gamma,\{p\}}^{a}(\vec{x})=X_{\beta}^{a}(\vec{x})
$$

and that 25) holds. For this reason we denote $\hat{H}_{\beta}$ by $\hat{H}_{\gamma,\{p\}}$.

Conversely, it can be checked that for any loop $\beta \in \mathcal{L}_{x_{0}}, X_{\beta}^{a}(\vec{x})=X_{\gamma,\{p\}}^{a}(\vec{x})$, where the closed, oriented graph $\gamma$ is the union of the edges which comprise $\beta$ with the orientations of the edges in $\gamma$ chosen arbitrarily and the labelling $\{q\}$, given such a choice of orientation is as follows. Let the number of times an edge $e_{i}$ is traversed in $\beta$, in the same direction as its orientation in $\gamma$, be $q_{1 i}$. Let the number of times the edge $e_{i}$ is traversed in $\beta$, in the opposite direction to its orientation in $\gamma$, be $q_{2 i}$. Then $e_{i}$ is labelled by $q_{i}=q_{1 i}-q_{2 i}$. Henceforth, we shall use the labelling of holonomies by their associated charge networks (i.e. $H_{\gamma,\{p\}}$ ) interchangabley with their labelling by loops (i.e. $\left.H_{\beta}\right)$. Thus, if there is no charge labelling in the subscript to $H$, the label is to be understood as a loop else as an associated charge network.

(4) Consider the holonomy operator associated with charge network label $(\alpha,\{q\})$. I Then $\hat{H}_{\alpha,\{q\}}$ maps $\mid \gamma,\{p\}>$ to a new charge network state based on the graph $\gamma \cup \alpha$ consisting of the union of the sets of edges belonging to $\gamma$ and $\alpha$. The edges of $\gamma \cup \alpha$ are oriented and labelled with charges as follows. Edges which are not shared by $\gamma$ and $\alpha$ retain their orientations and charge labels. Any shared edge labelled by the charge $p$ in $\gamma$ retains its orientation from $\gamma$ and has charge $p+q$ if it has the same

\footnotetext{
${ }^{4}$ Strictly speaking the discussion should and can be framed in terms of holonomically equivalent labels (i.e. $(\alpha,\{p\})$ is equivalent to $(\beta,\{q\})$ iff $\left.X_{\alpha,\{p\}}^{a}=X_{\beta,\{q\}}^{a}\right)$. We gloss over this subtlety in the interest of pedagogy.

${ }^{5} \mathrm{It}$ is assumed that edges of $\alpha, \gamma$ overlap only if they are identical and that intersections of $\alpha, \gamma$ occur only at vertices of $\alpha, \gamma$. This entails no loss of generality, since we can always find graphs which are holonomically equivalent to $\alpha, \gamma$ and for which the assumption holds.
} 
orientation in $\alpha$ and charge $p-q$ if it has opposite orientation in $\alpha$. We denote this new state by $\mid \gamma \cup \alpha,\{p \cup q\}>$. Thus

$$
\hat{H}_{\alpha,\{q\}}|\gamma,\{p\}>=| \gamma \cup \alpha,\{p \cup q\}>
$$

It can be checked that (20), (24) and (28) define a representation of the Poisson bracket algebra of equation( $(9)$ such that $\hat{H}_{\gamma}$ is unitary and $\hat{E}_{r}^{a}(\vec{x})$ is self adjoint.

Next, we review the construction of the 'dual' representation on the space of algebraic duals. Let $\mathcal{D} \subset L^{2}\left(\overline{\mathcal{A} / \mathcal{G}}, d \mu_{0}\right)$ be the (dense) set of finite linear combinations of charge network states. Let $\mathcal{D}^{*}$ be the space of algebraic duals to $\mathcal{D}$ i.e. every $\Phi \in \mathcal{D}^{*}$ is a complex linear map on $\mathcal{D}$. Define the action of $\hat{E}_{r}^{a}(\vec{x}), \hat{H}_{\alpha}$ on $\mathcal{D}^{*}$ through

$$
\begin{aligned}
\left(\hat{E}_{r}^{a}(\vec{x}) \Phi\right)(\mid \gamma,\{p\}>) & :=\Phi\left(\hat{E}_{r}^{a}(\vec{x})^{\dagger} \mid \gamma,\{p\}>\right)=\Phi\left(\hat{E}_{r}^{a}(\vec{x}) \mid \gamma,\{p\}>\right) \\
\left(\hat{H}_{\alpha} \Phi\right)(\mid \gamma,\{p\}>) & :=\Phi\left(\hat{H}_{\alpha}^{\dagger} \mid \gamma,\{p\}>\right)=\Phi\left(\hat{H}_{\alpha^{-1}} \mid \gamma,\{p\}>\right)
\end{aligned}
$$

Equations (29) and (30) provide an (anti-)representation of the Poisson bracket algebra (9). Note that $\mathcal{D}^{*}$ merely provides a linear representation space for the dual representation - it does not inherit any natural inner product from $\mathcal{D}$.

In section 3.2 we shall see that the natural arena to discuss the relation between the $r$-Fock representation and the qef representation is the space of algebraic duals, $\mathcal{D}^{*}$.

\subsection{The condition of Poincare invariance}

Even in linear quantum field theory there is no analog of the Stone- von Neuman uniqueness theorem for quantum mechanics on a vector space. Hence there are infinitely many inequivalent representations of the Poisson bracket algebra of smeared holonomies (see equation (6) ). The Fock space representation is singled out by the additional requirement of Poincare invariance, which in turn, is encoded in the specific choice of complex structure (i.e. the positive-negative frequency decomposition) for the Fock representation. This choice is equivalent to the requirement that the Fock vacuum be a zero eigenstate of the Fock space annihilation operators. In section 3.2.1 we shall express this requirement as a relation between the action of the smeared holonomy and electric field operators on the Fock vacuum (see equation (37)). 
The image of this relation in the $r$-Fock representation (see equation (38)) may be thought of as a condition which picks out the $r$-Fock vacuum. In other words, the $r$-Fock vacuum may be thought of as a solution to the condition (38). Since this condition relates the action of the holonomy and the smeared electric field operators, it is well defined in any representation of the Poisson bracket algebra of equation (9). In section 3.2.2 we impose the condition (38) in the (dual-)qef representation and show that it has a unique (upto a multiplicative constant) solution! Since this condition is deduced from the requirement of Poincare invariance, we may interpret the solution of (38) as the $r$-Fock vacuum expressed as a state in the (dual-)qef representation.

\subsubsection{Poincare invariance in terms of smeared holonomies}

Our conventions for the Fock space representation are as follows. The expansions of the field operators in Coulomb gauge are

$$
\begin{aligned}
\hat{A}_{a}(\vec{x}) & =\frac{1}{q_{0}(2 \pi)^{\frac{3}{2}}} \int \frac{d^{3} k}{\sqrt{k}}\left(e^{i \vec{k} \cdot \vec{x}} \frac{\hat{a}_{a}(\vec{k})}{\sqrt{2}}+\text { hermitian conjugate }\right) \\
\hat{E}_{a}(\vec{x}) & =\frac{1}{(2 \pi)^{\frac{3}{2}}} \int d^{3} k \sqrt{k}\left(-i e^{i \vec{k} \cdot \vec{x}} \frac{\hat{a}_{a}(\vec{k})}{\sqrt{2}}+\text { hermitian conjugate }\right) \\
\Rightarrow \hat{H}_{\alpha,\{q\}_{(r)}} & =\exp \left(\frac{i}{q_{0}} \int \frac{d^{3} k}{\sqrt{2 k}} X_{\alpha,\{q\}_{(r)}}^{a}(\vec{k})\left(\hat{a}_{a}(\vec{k})+\hat{a}_{a}^{\dagger}(\vec{k})\right)\right)
\end{aligned}
$$

The commutation relation between the annihilation and creation operators is

$$
\left[\hat{a}_{a}(\vec{k}), \hat{a}_{b}^{\dagger}(\vec{l})\right]=\left(\delta_{a b}-\frac{k_{a} k_{b}}{k^{2}}\right) \delta(\vec{k}, \vec{l})
$$

Using these equations, the vacuum expectation value of the smeared holonomy operator (also called, in the language of [18], 'the Fock Positive Linear Functional' or the 
Fock PLF and denoted by $\Gamma_{F}$ ) evaluates to

$$
\Gamma_{F}([\alpha,\{q\}]):=<0\left|H_{\alpha,\{q\}_{(r)}}\right| 0>=\exp \left(-\frac{1}{4 q_{0}^{2}} \int \frac{d^{3} k}{k}\left|X_{\alpha,\{q\}_{(r)}}^{a}(\vec{k})\right|^{2}\right)
$$

With equation (34), equations (32) and (33) provide a representation of the Poisson bracket algebra (6). As discussed above the condition of Poincare invariance implies

$$
\hat{a}_{a}(\vec{k}) \mid 0>=0
$$

This, in turn, implies the following relation between the smeared holonomy and the electric field operators:

$$
\left(\Gamma_{F}([\alpha,\{q\}])\right)^{2} e^{-\int \frac{d^{3} x}{q_{0}}\left(\frac{1}{\sqrt{-\left(\partial^{c} \partial_{c}\right)}} X_{\alpha,\{q\}_{(r)}}^{a}(\vec{x})\right) \hat{E}_{a}(\vec{x})}\left|0>=\hat{H}_{\alpha,\{q\}_{(r)}}^{\dagger}\right| 0>.
$$

This equation holds for every closed oriented graph $\alpha$ and encodes the condition of Poincare invariance in terms of elements of the algebra of smeared holonomies and the electric field.

\subsubsection{Poincare invariance in terms of holonomies}

The image of condition (37) in the $r$-Fock representation is

$$
\left(\Gamma_{F}([\alpha,\{q\}])\right)^{2} e^{-\int \frac{d^{3} x}{q_{0}}\left(\frac{1}{\sqrt{-\left(\partial^{c} \partial_{c}\right)}} X_{\alpha,\{q\}}^{a}(\vec{x})\right) \hat{E}_{r a}(\vec{x})}\left|0_{r}>=\hat{H}_{\alpha,\{q\}}^{\dagger}\right| 0_{r}>.
$$

We impose this condition in the qef representation on the space $\mathcal{D}^{*}$ of algebraic duals defined in section 3.1. Thus, the following equation is to be solved for some $\Phi_{0} \in \mathcal{D}^{*}:$

$$
\left(\Gamma_{F}([\alpha])\right)^{2} e^{-\int \frac{d^{3} x}{q_{0}}\left(\frac{1}{\sqrt{-\left(\partial^{c} \partial_{c}\right)}} X_{\alpha,\{q\}}^{a}(r)(\vec{x})\right) \hat{E}_{r a}(\vec{x})} \Phi_{0}=\hat{H}_{\alpha,\{q\}}^{\dagger} \Phi_{0} .
$$

Any element of $\mathcal{D}^{*}$ can be written as a formal sum over all charge network states as follows. If $\Phi_{0}(\mid \gamma,\{p\}>)=c_{\gamma,\{p\}}$ where $c_{\gamma,\{p\}}$ is a complex number, it follows that $\Phi_{0}$ can be written as

$$
\Phi_{0}=\sum_{\gamma,\{p\}} c_{\gamma,\{p\}}<\gamma,\{p\} \mid .
$$

\footnotetext{
${ }^{6}$ Note that the expression for $\Gamma_{F}$ in $[18$ is incorrect. The correct expression is (35) above and differs from the expression in [18 by a factor in its exponent. Also, in 18] the parameter $q_{0}^{-1}$ was written as $e$, but factors of $e$ appeared in $[18]$ often in the wrong places. We have corrected the erroneous expressions of [18] in this work. With appropriate corrections regarding these factors of $q_{0}$, all the results of [18] continue to hold.
} 
Substitution of this in (39) and projection of the resulting equation onto the ket $\mid \beta,\{t\}>$ yields

$$
\begin{aligned}
\left(\Gamma_{F}([\alpha,\{q\}])\right)^{2} \sum_{\gamma,\{p\}} c_{\gamma,\{p\}}<\gamma,\{p\}\left|e^{-\int \frac{d^{3} x}{q_{0}}\left(\frac{1}{\sqrt{-\left(\partial^{c} \partial_{c}\right)}} X_{\alpha,\{q\}}^{a}(\vec{x})\right) \hat{E}_{r a}(\vec{x})}\right| \beta,\{t\}> \\
=\sum_{\gamma,\{p\}} c_{\gamma,\{p\}}<\gamma,\{p\}\left|\hat{H}_{\alpha,\{q\}}\right| \beta,\{t\}>
\end{aligned}
$$

Orthonormality of the charge network states, together with (24) and (28), implies that

$$
\left(\Gamma_{F}([\alpha,\{q\}])\right)^{2} e^{-\int \frac{d^{3} x}{q_{0}^{2}}\left(\frac{1}{\sqrt{-\left(\partial^{c} \partial_{c}\right)}} X_{\alpha,\{q\}_{(r)}}^{a}(\vec{x})\right) X_{a \beta,\{t\}_{(r)}}(\vec{x})} c_{\beta,\{t\}}=c_{\beta \cup \alpha,\{t \cup q\}}
$$

This equation can now be solved for the coefficients $c_{\gamma,\{p\}}$; of course, since the equation is linear and homogeneous the solution will be ambiguous by an overall constant. We fix this ambiguity by setting the coefficient labelled by the trivial graph $\gamma=0, \overrightarrow{0}(s)=\vec{x}_{0}$, to be unity. Then setting $\beta=0$ in (42) yields

$$
c_{\alpha,\{q\}}=\left(\Gamma_{F}([\alpha,\{q\}])\right)^{2}
$$

for every charge network $\mid \alpha,\{q\}>$. It may be verified that, miraculously, this also provides a solution to (42)! Thus

$$
\Phi_{0}=\sum_{\gamma,\{p\}}\left(\Gamma_{F}([\gamma,\{p\}])\right)^{2}<\gamma,\{p\} \mid
$$

is the unique (upto an overall constant) solution to the condition (39)! We identify $\Phi_{0}$ as the state corresponding to the $r$-Fock vacuum.

As shown in 18 the action of the smeared holonomy operators on the Fock vacuum generates a dense subset of the Fock space. It follows that the action of the holonomy operators on the $r$-Fock vacuum generates a dense set of the $r$-Fock space. Therefore, we can use the dual representation of the holonomy operator (see equation (30)) on $\mathcal{D}^{*}$ to generate the corresponding set of states from $\Phi_{0}$. Call this set $\mathcal{L}^{*}$. Thus any element of $\mathcal{L}^{*}$ is of the form $\sum_{I=1}^{N} a_{I} \hat{H}_{\gamma^{I},\left\{p^{I}\right\}} \Phi_{0}$ for some complex $a_{I}$ and $N$ finite. 
As noted earlier, $\mathcal{D}^{*}$ (and hence $\mathcal{L}^{*}$ ) is not equipped with an inner product. Therefore an inner product on $\mathcal{L}^{*}$ must be chosen which implements the classical 'reality conditions', namely $H_{\gamma,\{p\}}^{*}=H_{\gamma,\{-p\}}$ and $E_{r}(\vec{x})^{*}=E_{r}(\vec{x})$, 0 on the corresponding quantum operators. It can be verified that the following inner product (naturally extendible to all of $\mathcal{L}^{*}$ ) implements the reality conditions:

$$
\left(\hat{H}_{\alpha,\{p\}} \Phi_{0}, \hat{H}_{\beta,\{q\}} \Phi_{0}\right)=\exp \left(-\frac{1}{4 q_{0}^{2}} \int \frac{d^{3} k}{k}\left|X_{\alpha \cup \beta,\{-p \cup q\}_{(r)}}^{a}(\vec{k})\right|^{2}\right) .
$$

It follows that the Cauchy completion of $\mathcal{L}^{*}$ with respect to this inner product results in a Hilbert space which can be identified as $r$-Fock space and that the representation given by equations (29) and (30) is exactly the $r$-Fock representation.

\section{Concluding remarks}

In this work we have related the diffeomorphism invariant, non-seperable quantized electric flux representation for quantum $U(1)$ theory to its standard Poincare invariant Fock space representation. This relation is based on the fact that the $U(1)$ holonomies play an important role in the construction of the qef representation.

Since the holonomy operators are well defined in the qef representation but not in the Fock representation, we first constructed a 1 parameter family of representations in which the holonomy operators are well defined and which are physically indistinguishable from the standard Fock representation. More precisely, the new representations are labelled by a positive parameter, $r$, with dimensions of length. For finite accuracy measurements at distance scales much larger than $r$, these ' $r$-Fock representations' are indistinguishable from the standard Fock representation.

Next, we related the $r$-Fock representation (for any fixed $r$ ) to the qef representation by identifying the $r$-Fock vacuum as a (distributional) state in the (dual) qef representation. This identification was achieved by solving, in the qef representation, equation (39) inspired by Poincare invariance, which enforced the condition that the

\footnotetext{
7 Rather than using these reality conditions directly for the operator $\hat{E}_{r}(\vec{x})$, it is simpler to use

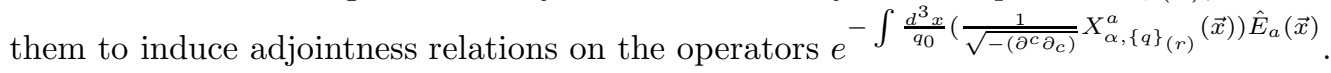


annihilation operator of the $r$-Fock representation kill its vacuum state. The qef representation is built on the property of diffeomorphism invariance and 'knows' nothing about Poincare invariance and hence we find it truly remarkable that the condition (39) which arises from Poincare invariance of the Fock vacuum can be solved essentially uniquely in the (dual) qef representation. Once the $r$ - Fock vacuum was identified as a state in the dual qef representation, we constructed states corresponding to a dense set in $r$-Fock space by the (dual) action of the holonomy operators on the $r$-Fock vacuum. Finally, the inner product was obtained on this set of states by requiring that the classical reality conditions be implemented as adjointness conditions on the corresponding quantum operators. Thus, in the qualitative language of the introduction section, we may say that non-normalizable infinite superpositions of 1 dimensional, polymer like excitations conspire, in collusion with the inner product (45), to acquire the character of $3 d$ wavelike excitations in Fock space.

From the point of view of quantum gravity, we think that we have unravelled an important set of structures which will help relate the Fock space of gravitons of linearised gravity to appropriate semiclassical states in loop quantum gravity. In loop quantum gravity also, it is the dual representation to the kinematic spin network representation which serves as the 'home' for physical, dynamically relevant quantum states of the gravitational field. Although there is no consensus on the exact physical states of the theory, it is still true that the structure of the physical states is that of distributions on the finite span of spin network states and that quantum operators act on these distributions via dual action. Further, the issue of the correct inner product on the space of physical states is still open and this inner product may have very different properties from the kinematical one in the context of solutions to the Hamiltonian constraint. Since the $r$-Fock representation for $U(1)$ theory has been obtained, in this work, as the dual representation on distributions to the finite span of charge network states with the 'physical' inner product (45) unrelated to the 'kinematic' inner product (20), we feel that our results will play an important role in relating gravitons to physical states in loop quantum gravity. In the loop quantum gravity case there are other complications such as the 'linearization' of the 
non abelian gauge group to 3 copies of $U(1)$ [19], as well as the identification of a state corresponding to flat spacetime. These issues are currently under investigation.

Apart from potential applications to quantum gravity, it would be of interest to understand the $r$-Fock representations in their own right. In this regard we raised the question in [18] as to whether the $r$-Fock representation could be realised as an $L^{2}\left(\overline{\mathcal{A} / \mathcal{G}}, d \mu_{F(r)}\right)$ representation for some measure $d \mu_{F(r)}$ on $\overline{\mathcal{A} / \mathcal{G}}$. As we show in the appendix, the answer to this question is in the affirmative and it would be of interest to understand the properties of this new $r$-Fock measure. Whether these new representations have applications outside of loop quantum gravity remains to be seen.

\section{Acknowledgments}

I gratefully acknowledge helpful discussions of this material with Abhay Ashtekar. I am indebted to Marcus van Bers for sharing his insights with me.

\section{Appendix}

\section{A1 Existence of the $r$-Fock measure on $\overline{\mathcal{A} / \mathcal{G}}$}

In what follows we shall freely use results and notation from [18 as well as from previous sections. If We shall be brief - the interested reader may work out the details. The $r$ - Fock measure exists on $\overline{\mathcal{A} / \mathcal{G}}$ iff the $r$-Fock positive linear functional,

$$
\Gamma_{F(r)}\left(\sum_{I=1}^{N} a_{I}\left[\alpha_{I}\right]\right)=\sum_{I=1}^{N} a_{I} \exp \left(-\frac{1}{4 q_{0}^{2}} \int \frac{d^{3} k}{k}\left|X_{\alpha_{I(r)}}^{a}(\vec{k})\right|^{2}\right)
$$

is continuous with respect to the $C^{*}$ norm, ||$\sum_{I=1}^{N} a_{I}\left[\alpha_{I}\right]||:=\sup _{A \in \mathcal{A}}\left|\sum_{I=1}^{N} a_{I} H_{\alpha_{I}}(A)\right|$. From [18] we have that

$$
\left\|\sum_{i=1}^{N} a_{I}\left[\alpha_{I}\right]\right\|=\sup _{A \in \mathcal{A}}\left|\sum_{I=1}^{N} a_{I} H_{\alpha_{I(r)}}(A)\right| .
$$

\footnotetext{
${ }^{8}$ We shall use the corrected expressions of [18] - see footnote 6 in this regard.
} 
Also, from (10) we have that

$$
\Gamma_{F(r)}\left(\sum_{I=1}^{N} a_{I}\left[\alpha_{I}\right]\right)=<0\left|\sum_{I=1}^{N} a_{I} \hat{H}_{\alpha_{I(r)}}\right| 0>
$$

Using the standard $L^{2}\left(\mathcal{S}^{*}, d \mu_{G}\right)$ [18, 21] representation of Fock space where $\mathcal{S}^{*}$ is an appropriate space of tempered distributions and $d \mu_{G}$ is the standard, unit volume, Gaussian measure, we have

$$
\begin{aligned}
<0\left|\sum_{I=1}^{N} a_{I} \hat{H}_{\alpha_{I(r)}}\right| 0> & =\int_{A_{a} \in \mathcal{S}^{*}} d \mu_{G}(A) \sum_{I=1}^{N} a_{I} H_{\alpha_{I(r)}}(A) \\
\Rightarrow|<0| \sum_{I=1}^{N} a_{I} \hat{H}_{\alpha_{I(r)}}|0>| & \leq \sup _{A \in \mathcal{S}^{*}}\left|\sum_{I=1}^{N} a_{I} H_{\alpha_{I(r)}}(A)\right| \int_{A_{a} \in \mathcal{S}^{*}} d \mu_{G}(A) \\
& =\sup _{A \in \mathcal{S}^{*}}\left|\sum_{I=1}^{N} a_{I} H_{\alpha_{I(r)}}(A)\right| .
\end{aligned}
$$

Since $X_{\alpha_{(r)}}^{a}(\vec{x})$ is in Schwartz space, it follows that every $A_{a} \in \mathcal{S}^{*}$ defines a homeomorphism, $h$, from $\mathcal{H G}_{r}$ to $U(1)$ (the element of $U(1)$ corresponding to a loop $\alpha$ is just $\exp i \int_{R^{3}} X_{\alpha_{(r)}}^{a}(\vec{x}) A_{a}(\vec{x}) d^{3} x$ ). It follows from the considerations of 18 (see especially equation $(\mathrm{A} 15)$ of [18]) that

$$
\sup _{A \in \mathcal{S}^{*}}\left|\sum_{I=1}^{N} a_{I} H_{\alpha_{I(r)}}(A)\right| \leq \sup _{A \in \mathcal{A}}\left|\sum_{I=1}^{N} a_{I} H_{\alpha_{I(r)}}(A)\right| .
$$

It follows from (47), (48), (50) and (51) that

$$
\left|\Gamma_{F(r)}\left(\sum_{I=1}^{N} a_{I}\left[\alpha_{I}\right]\right)\right| \leq\left\|\sum_{i=1}^{N} a_{I}\left[\alpha_{I}\right]\right\| .
$$

This implies that $\Gamma_{F(r)}$ is continuous with respect to \|\| and hence that an $r$-Fock measure, $d \mu_{F(r)}$ exists on $\overline{\mathcal{A} / \mathcal{G}}$. 


\section{References}

[1] T. A. Schilling, 'Bibliography OF publications related to Classical and Quantum Gravity in terms of the Ashtekar variables', eprint gr-qc/9409031.

[2] C. Rovelli, 'Loop Quantum Gravity', Living Rev.Rel.1:1,1998.

[3] A. Ashtekar, Phys.Rev.D36, 1587 (1987).

[4] J.Fernando Barbero, Phys.Rev.D51, 5507 (1995).

[5] C. Rovelli and L. Smolin, Nucl. Phys. B331, 80 (1990).

[6] T. Jacobson and L. Smolin, Nucl.Phys.B299, 295 (1988).

[7] A. Ashtekar and J. Lewandowski in Quantum Gravity and Knots ed. by J. Baez (Oxford Univ. Press).

[8] A. Ashtekar and C. J. Isham, Class. Quantum Grav. 9, 1433 (1992) .

[9] D. Marolf and J. Mourão, Commun. Math. Phys. ,583 (1995).

[10] J. Baez, Lett. Math. Phys.31, 213 (1994).

[11] A. Ashtekar and J. Lewandowski, J. Geom. Phys.17, 19 (1995).

[12] C. Rovelli and L. Smolin, Phys.Rev.D52, 5743 (1995).

[13] J. Baez, in The interface of knots and physics (San Francisco, 1995) 167-203, e-Print Archive: gr-qc/9504036

[14] A. Ashtekar, J. Lewandowski, D. Marolf, J. Mourão and T. Thiemann, J. Math. Phys. 36, 6456 (1995).

[15] T. Thiemann, Class.Quant.Grav.15, 839 (1998).

[16] A. Corichi and K. V. Krasnov, Mod.Phys.Lett.A13, 1339 (1998).

[17] A. Ashtekar and C. J. Isham, Phys. Lett.B274, 393 (1992). 
[18] M. Varadarajan, Phys.Rev.D61, 104001 (2000).

[19] A. Ashtekar, C. Rovelli and L. Smolin, Phys. Rev.D44 (1991), 1740.

[20] A. Ashtekar and J. Lewandowski, Class.Quant.Grav.14, A55 (1997).

[21] J. Glimm and A. Jaffe, Quantum Physics, (Springer-Verlag, New York, 1987). 